Please do not remove this page

RMIT

UNIVERSITY

\title{
Reasoning about agent programs using ATL-like logics
}

Yadav, Nitin; Sardina, Sebastian

https://researchrepository.rmit.edu.au/esploro/outputs/9921859166601341/filesAndLinks?institution=61RMIT_INST\&index=null

Yadav, N., \& Sardina, S. (2012). Reasoning about agent programs using ATL-like logics. Lecture Notes in Computer Science, 7519, 437-449.

https://researchrepository.rmit.edu.au/discovery/fulldisplay/alma9921859166601341/61RMIT_INST:Resea rchRepository

Document Version: Accepted Manuscript

Repository homepage: https://researchrepository.rmit.edu.au

(c) Springer-Verlag Berlin Heidelberg 2012

Downloaded On 2023/04/26 18:57:28 +1000

Please do not remove this page 
Thank you for downloading this document from the RMIT Research Repository.

The RMIT Research Repository is an open access database showcasing the research outputs of RMIT University researchers.

RMIT Research Repository: http://researchbank.rmit.edu.au/

\section{Citation:}

Yadav, N and Sardina, S 2012, 'Reasoning about agent programs using ATL-like logics', Lecture Notes in Computer Science, vol. 7519, pp. 437-449.

See this record in the RMIT Research Repository at:

http://researchbank.rmit.edu.au/view/rmit:20473

Version: Accepted Manuscript

Copyright Statement: (c) Springer-Verlag Berlin Heidelberg 2012

Link to Published Version:

http://link.springer.com/content/pdf/10.1007\%2F978-3-642-33353-8_34 


\title{
Reasoning about Agent Programs using ATL-like Logics
}

\author{
Nitin Yadav and Sebastian Sardina * \\ RMIT University, Melbourne, Australia.
}

\begin{abstract}
We propose a variant of Alternating-time Temporal Logic (ATL) grounded in the agents' operational know-how, as defined by their libraries of abstract plans. Inspired by ATLES, a variant itself of ATL, it is possible in our logic to explicitly refer to "rational" strategies for agents developed under the Belief-Desire-Intention agent programming paradigm. This allows us to express and verify properties of BDI systems using ATL-type logical frameworks.
\end{abstract}

Keywords: Agent Programming, Reactive plans, ATL, Model Checking.

\section{Introduction}

The formal verification of agent-oriented programs requires logic frameworks capable of representing and reasoning about agents' abilities and capabilities, and the goals they can feasibly achieve. In particular, we are interested here in programs written in the family of Belief-Desire-Intention (BDI) agent programming systems [5, 6, 18], a popular paradigm for building multi-agent systems. Traditional BDI logics based on CTL (e.g., [17]) are generally too weak for representing ability; their success has primarily been in defining "rationality postulates," i.e., constraints on rational behaviour. Further, such logics do not encode agents' capabilities (as represented by their plan libraries) and thereby leave a sizable gap between agent programs and their formal verification.

Recent work (e.g., [1, 2, 9]) has better bridged the gap between formal logic and practical programming by providing an axiomatisation of a class of models that is designed to closely model a programming framework. However, this is done by restricting the logic's models to those that satisfy the transition relations of agents' plans, as defined by the semantics of the programming language itself. In such a framework, it is not possible to reason about the agent's know-how and what the agent could achieve if it had specific capabilities. It is also not possible to reason about coalition of agents.

Our aim thus is to define a framework, together with model checking techniques, that will allow us to speculate about a group of agents' capabilities and what they can achieve with such capabilities under the BDI paradigm, which enables abstract plans written by programmers to be combined and used in real-time under the principles of

This requires the ability to represent capabilities directly in our logic. To that end, we adapt ATLES, a version of ATL (Alternating-time Temporal Logic) [3] with Explicit Strategies [20], to our purpose. ATL is a logic for reasoning about the ability of agent coalitions in multi-player game structures. This is achieved by reasoning about

\footnotetext{
* We would like to thank Lawrence Cavedon for earlier discussions on the topic of this paper. We acknowledge the support of the Australian Research Council under grant DP120100332.
} 
strategies (and their success) employed by teams of agents: $\langle\langle A\rangle\rangle \varphi$ expresses that the coalition team of agents $A$ has a joint strategy for guaranteeing that the temporal property $\varphi$ holds. Walther et al. [20], standard ATL does not allow agents' strategies to be explicitly represented in the syntax of the logic. They thus rectified this shortcoming by defining ATLES, which extends ATL by allowing strategy terms in the language: $\left\langle\langle A\rangle_{\rho} \varphi\right.$ holds if coalition $A$ has a joint strategy for ensuring $\varphi$, when some agents are committed to specific strategies as specified by so-called commitment function $\rho$.

In this paper, we go further and develop a framework-called BDI-ATLES-in which the strategy terms are tied directly to the plans available to agents under the notion of practical reasoning embodied by the BDI paradigm [6, 18]: the only strategies that can be employed by a BDI agent are those that ensue by the (rational) execution of its predefined plans, given its goals and beliefs. The key construct $\left\langle\langle A\rangle_{\omega, \varrho} \varphi\right.$ in the new framework states that coalition $A$ has a joint strategy for ensuring $\varphi$, under the assumptions that some agents in the system are BDI-style agents with capabilities and goals as specified by assignments $\omega$ and $\varrho$, respectively. For instance, in the Gold Mining domain from the International Agent Contest, ${ }^{1}$ one may want to verify if two miner agents programmed in a BDI language can successfully collect gold pieces when equipped with navigation and communication capabilities and want to win the game, while the opponent agents can perform any physically legal action. More interesting, a formula like $\left\langle\langle A\rangle_{\emptyset, \emptyset} \varphi \supset\left\langle\langle A\rangle_{\omega, \varrho} \varphi\right.\right.$ can be used to check whether coalition $A$ has enough know-how and motivations to carry out a task $\varphi$ that is indeed physically feasible for the coalition.

We observe that the notion of "rationality" used in this work is that found in the literature on BDI and agent programming, rather than that common in game-theory (generally captured via solution concepts). As such, rationality shall refer from now on to reasonable constraints on how the various mental modalities-e.g., beliefs, intention, goals-may interact. In particular, we focus on the constraint that agents select actions from their know-how in order to achieve their goals in the context of their beliefs.

Finally, we stress that this work aims to contribute to the agent-oriented programing community more than to the (ATL) verification one. Indeed, our aim is to motivate the former to adopt well-established techniques in game-theory for the effective verification of their "reactive" style agent programs.

\section{Preliminaries}

\subsection{ATL/ATLES Logics of Coalitions}

Alternating-time Temporal Logic (ATL) [3] is a logic for reasoning about the ability of agent coalitions in multi-agent game structures. ATL formulae are built by combining propositional formulas, the usual temporal operators-namely, $\bigcirc$ ("in the next state"), $\square$ ("always"), $\diamond$ ("eventually"), and $\mathcal{U}$ ("strict until")—and a coalition path quantifier $\langle\langle A\rangle\rangle$ taking a set of agents $A$ as parameter. As in CTL, which ATL extends, temporal operators and path quantifiers are required to alternate. Intuitively, an ATL formula $\langle\langle A\rangle\rangle \phi$, where $A$ is a set of agents, holds in an ATL structure if by suitably choosing their moves, the agents in $A$ can force $\phi$ true, no matter how other agents happen to

\footnotetext{
${ }^{1}$ http://www.multiagentcontest.org/
} 
move. The semantics of ATL is defined in so-called concurrent game structures where, at each point, all agents simultaneously choose their moves from a finite set, and the next state deterministically depends on such choices. More concretely, an ATL structure is a tuple $\mathcal{M}=\langle\mathcal{A}, Q, \mathcal{P}, A c t, d, \mathcal{V}, \sigma\rangle$, where $\mathcal{A}=\{1, \ldots, k\}$ is a finite set of agents, $Q$ is the finite set of states, $\mathcal{P}$ is the finite set of propositions, Act is the set of all domain actions, $d: \mathcal{A} \times Q \mapsto 2^{A c t}$ indicates all available actions for an agent in a state, $\mathcal{V}: Q \mapsto 2^{\mathcal{P}}$ is the valuation function stating what is true in each state, and $\sigma: Q \times A c t^{|\mathcal{A}|} \mapsto Q$ is the transition function mapping a state $q$ and a joint-move $a \in \mathcal{D}(q)$-where $\mathcal{D}(q)=\times_{i=1}^{|\mathcal{A}|} d(i, q)$ is the set of legal joint-moves in $q$-to the resulting next state $q^{\prime}$.

A path $\lambda=q_{0} q_{1} \cdots$ in a structure $\mathcal{M}$ is a, possibly infinite, sequence of states such that for each $i \geq 0$, there exists a joint-move $a_{\boldsymbol{i}} \in \mathcal{D}\left(q_{i}\right)$ for which $\sigma\left(q_{i}, a_{\boldsymbol{i}}\right)=q_{i+1}$. We use $\lambda[i]=q_{i}$ to denote the $i$-th state of $\lambda, \Lambda$ to denote the set of all paths in $\mathcal{M}$, and $\Lambda(q)$ to denote those starting in $q$. Also, $|\lambda|$ denotes the length of $\lambda$ as the number of state transitions in $\lambda:|\lambda|=\ell$ if $\lambda=q_{0} q_{1} \ldots q_{\ell}$, and $|\lambda|=\infty$ if $\lambda$ is infinite. When $0 \leq i \leq j \leq|\lambda|$, then $\lambda[i, j]=q_{i} q_{i+1} \ldots q_{j}$ is the finite subpath between the $i$-th and $j$-th steps in $\lambda$. Finally, a computation path in $\mathcal{M}$ is an infinite path in $\Lambda$.

To provide semantics to formulas $\langle\langle\cdot\rangle\rangle$, ATL relies on the notion of agent strategies. Technically, an ATL strategy for an agent agt is a function $f_{\text {agt }}: Q^{+} \mapsto A c t$, where $f_{a g t}(\lambda q) \in d(a g t, q)$ for all $\lambda q \in Q^{+}$, stating a particular action choice of agent agt at path $\lambda q$. A collective strategy for group of agents $A \subseteq \mathcal{A}$ is a set of strategies $F_{A}=$ $\left\{f_{\text {agt }} \mid\right.$ agt $\left.\in \mathcal{A}\right\}$ providing one specific strategy for each agent agt $\in A$. For a collective strategy $F_{A}$ and an initial state $q$, it is not difficult to define the set out $\left(q, F_{A}\right)$ of all possible outcomes of $F_{A}$ starting at state $q$ as the set of all computation paths that may ensue when the agents in $A$ behave as prescribed by $F_{A}$, and the remaining agents follow any arbitrary strategy $[3,20]$. The semantics for the coalition modality is then defined as follows (here $\phi$ is a path formula, that is, it is preceded by $\bigcirc$, $\square$, or $\mathcal{U}$, and $\mathcal{M}, \lambda \models \phi$ is defined in the usual way [3]):

$$
\begin{gathered}
\mathcal{M}, q \models\langle\langle A\rangle\rangle \phi \text { iff there is a collective strategy } F_{A} \text { such that for all computations } \\
\qquad \lambda \in \operatorname{out}\left(q, F_{A}\right) \text {, we have } \mathcal{M}, \lambda \models \phi .
\end{gathered}
$$

The coalition modality only allows for implicit (existential) quantification over strategies. In some contexts, though, it is important to refer to strategies explicitly in the language, e.g., can a player win the game if the opponent plays a specified strategy? To address this limitation, Walther et al. [20] proposed ATLES, an extension of ATL where the coalition modality is extended to $\langle\langle A\rangle\rangle_{\rho}$, where $\rho$ is a commitment function, that is, a partial function mapping agents to so-called strategy terms. Formula $\left\langle\langle A\rangle_{\rho} \phi\right.$ thus means that "while the agents in the domain of $\rho$ act according to their commitments, the coalition A can cooperate to ensure $\phi$ as an outcome."

The motivation for our work stems from the fact that ATLES is agnostic on the source of the strategic terms: all meaningful strategies have already been identified. In the context of multi-agent systems, it may not be an easy task to identify those strategies compatible with the agents' behaviors, as those systems are generally built using programming frameworks [5] that are very different from ATL(ES). 


\subsection{BDI Programming}

The BDI agent-oriented programming paradigm is a popular and successful approach for building agent systems, with roots in philosophical work on rational action [6] and a plethora of programming languages and systems available, such as JACK, JASON, JADEX, 2APL [5], and GOAL [11], among others.

A typical BDI agent continually tries to achieve its goals (or desires) by selecting an adequate plan from its plan library given its current beliefs, and placing it into the intention base for execution. The agent's plan library $\Pi$ encodes the standard operational knowledge of the domain by means of a set of plan-rules (or "recipes") of the form $\phi[\alpha] \psi$ : plan $\alpha$ is a reasonable plan to adopt for achieving $\psi$ when (context) condition $\phi$ is believed true. For example, walking towards location $x$ from $y$ is a reasonable strategy, if there is a short distance between $x$ and $y$ (and the agent wants to be eventually at location $x$ ). Conditions $\phi$ and $\psi$ are (propositional) formulas talking about the current and goal states, respectively. Though different BDI languages offer different constructs for crafting plans, most allow for sequences of domain actions that are meant to be directly executed in the world (e.g., lifting an aircraft's flaps), and the posting of (intermediate) sub-goals ! $\varphi$ (e.g., obtain landing permission) to be resolved. The intention base, in turn, contains the current, partially executed, plans that the agent has already committed to for achieving certain goals. Current intentions being executed provide a screen of admissibility for attention focus [6].

Though we do not present it here for lack of space, most BDI-style programming languages come with a clear single-step semantics basically realizing [18]'s execution model in which (rational) behavior arises due to the execution of plans from the agent's plan library so as to achieve certain goals relative to the agent's beliefs.

\section{BDI-ATLES: ATL for BDI Agents}

Here we develop an ATL(ES)-like logic that bridges the gap between verification frameworks and BDI agent-oriented programming languages. The overarching idea is for BDI programmers to be able to encode BDI applications in ATL in a principled manner.

Recall that ATL(ES) uses strategies to denote the agent's choices among possible actions. For a BDI agent these strategies are implicit in her know-how. In particular, we envision BDI agents defined with a set of goals and so-called capabilities [7, 16]. Generally speaking, a capability is a set/module of procedural knowledge (i.e., plans) for some functional requirement. An agent may have, for instance, the Navigate capability encoding all plans for navigating an environment. Equipped with a set of capabilities, a BDI agent executes actions as per plans available so as to achieve her goals, e.g., exploring the environment. In this context, the BDI developer is then interested in what agents can achieve at the level of goals and capabilities. Inspired by ATLES, we develop a logic that caters for this requirement without departing much from the ATL framework.

In this work, we shall consider plans consisting of single actions, that is, given BDI plan for the form $\phi[\alpha] \psi$, the body of the plan $\alpha$ consists of one primitive action. Such plans are akin to those in the GOAL agent programming language [11], as well as universal-plans [19], and reactive control modules [4]. Let $\boldsymbol{\Pi}_{\text {Act }}^{\mathcal{P}}$ be the (infinite) set of all possible plan-rules given a set of actions Act and a set of domain propositions $\mathcal{P}$. 


\subsection{BDI-ATLES Syntax}

The language of BDI-ATLES is defined over a finite set of atomic propositions $\mathcal{P}$, a finite set of agents $\mathcal{A}$, and a finite set of capability terms $\mathcal{C}$ available in the BDI application of concern. Intuitively, each capability term $c \in \mathcal{C}$ (e.g., Navigate) stands for a plan library $\Pi^{c}$ (e.g., $\Pi^{\text {Navigate }}$ ). As usual, a coalition is a set $A \subseteq \mathcal{A}$ of agents. A capability assignment $\omega$ consists of a set of pairs of agents with their capabilities of the form $\left\langle a g t: C_{a g t}\right\rangle$, where agt $\in \mathcal{A}$ and $C_{a g t} \subseteq \mathcal{C}$. A goal assignment $\varrho$, in turn, defines the goal base (i.e., set of propositional formulas) for some agents, and is a set of tuples of the form $\left\langle a g t: G_{a g t}\right\rangle$, where agt $\in \mathcal{A}$ and $G_{a g t}$ is a set of boolean formulas over $\mathcal{P}$. We use $\mathcal{A}_{\omega}$ to denote the set of agents for which their capabilities are defined by assignment $\omega$, that is, $\mathcal{A}_{\omega}=\left\{a g t \mid\left\langle a g t: C_{a g t}\right\rangle \in \omega\right\}$. Set $\mathcal{A}_{\varrho}$ is defined analogously.

The set of BDI-ATLES formulas is then exactly like that of ATL(ES), except that coalition formulas are now of the form $\left\langle\langle A\rangle_{\omega, \varrho} \varphi\right.$, where $\varphi$ is a path formula (i.e., it is preceded by $\bigcirc, \square$, or $\mathcal{U}$ ), $A$ is a coalition, and $\omega$ and $\varrho$ range over capability and goal assignments, respectively, such that $\mathcal{A}_{\omega}=\mathcal{A}_{\varrho}$. Its intended meaning is as follows:

$\left\langle\langle A\rangle_{\omega, \varrho} \varphi\right.$ expresses that coalition of agents $A$ can jointly force temporal condition $\varphi$ to hold when BDI agents in $\mathcal{A}_{\omega}$ (or $\mathcal{A}_{\varrho}$, since $\mathcal{A}_{\varrho}=\mathcal{A}_{\omega}$ ) are equipped with capabilities as per assignment $\omega$ and (initial) goals are per assignment $\varrho$.

Notice that we require, in each coalition (sub)formula, that the agents for which capabilities and goals are assigned to be the same. This enforces the constraint that BDIstyle agents have both plans and goals. Hence, a formula of the form $\langle\langle A\rangle\rangle_{\emptyset,\left\{\left\langle a_{1}:\{\gamma\}\right\rangle\right\}} \varphi$ would not be valid, as agent $a_{1}$ has one goal (namely, to bring about $\gamma$ ), but its set of plans is not defined-we cannot specify what its rational behavior may be. This contrasts with formula $\langle\langle A\rangle\rangle_{\left\{\left\langle a_{1}: \emptyset\right\rangle\right\},\left\{\left\langle a_{1}:\{\gamma\}\right\rangle\right\}} \varphi$, a valid formula in which agent $a_{1}$ is assumed to have no plans (i.e., agent has empty know-how) and one goal.

Example 1. Consider the following simplified instance of the gold mining domain with three locations $A, B$ and $C$, a gold piece $\diamond$ at location $C$, the depot located at $B$ (rectangle location), and two players $\mathrm{Ag}$ (BDI agent) and En (enemy):

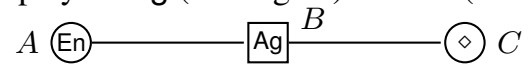

Players can move LEFT/RIGHT, PICK/DROP gold, or remain still by executing special action NOOP. Proposition $X_{Y}$, where $X \in\{\mathrm{Ag}, \mathrm{En}\}$ and $Y \in\{A, B, C\}$, encodes that player $X$ is at location $Y$; whereas propositions $G_{A}, G_{B}, G_{C}, G_{\mathrm{Ag}}$, and $G_{\mathrm{En}}$ denote that the gold is at location $A / B / C$ or being held by agent $A g / E n$, respectively. The depot is assumed to be always at $B$ and hence is not represented explicitly.

The winning condition for player $\mathrm{Ag}$ is $\psi_{W I N}=G_{B} \wedge \mathrm{Ag}_{B}$ : the player wins when collocated with gold at the depot.

Among the many capabilities available encoding the know-how information of the domain, we consider the following three. The Collect capability includes plans to pick gold, such as $\mathrm{Ag}_{C} \wedge G_{C}[\mathrm{PICK}] G_{B}$ : if gold needs to be at $B$ and agent is at $C$, where there is indeed gold, then execute the PICK action. Similarly, capability Deposit contains plans like $G_{\mathrm{Ag}} \wedge \mathrm{Ag}_{B}[\mathrm{DROP}] G_{B}$, for example, to allow dropping of gold at the desired location. Lastly, capability Navigate has plans for moving around, such as $\mathrm{Ag}_{C}[\mathrm{LEFT}] \mathrm{Ag}_{B}$ to move left from location $C$ to (desired destination) $B$. 


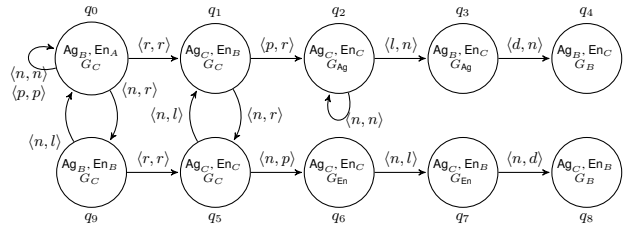

(a) A section of the BDI-ATLES alternating model.

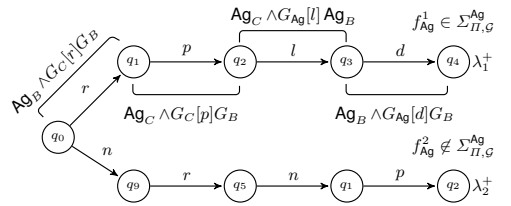

(b) Traces $\lambda_{1}^{+}$and $\lambda_{2}^{+}$resultant from strategies $f_{\mathrm{Ag}}^{1}$ and $f_{\mathrm{Ag}}^{2}$, respectively

Fig. 1. A fragment of a Gold domain model and a picture showing rational traces and strategies. Actions LEFT, RIGHT, PICK, DROP, and NOOP are abbreviated with their first letter.

The remaining of the section involves providing the right interpretation to such formulas, under the assumption that agents act rationally as per the BDI paradigm.

\subsection{BDI-ATLES Semantics}

A BDI-ATLES concurrent game structure is a tuple $\mathcal{M}=\langle\mathcal{A}, Q, \mathcal{P}, A c t, d, \mathcal{V}, \sigma, \Theta\rangle$, with:

- $\mathcal{A}, Q, \mathcal{P}, A c t, d, \mathcal{V}$ and $\sigma$ are as in ATL(ES).

- There is a distinguished dummy action NOOP $\in$ Act such that NOOP $\in d_{\text {agt }}(q)$ and $\sigma(q,\langle$ NOOP,$\ldots$, NOOP $\rangle)=q$, for all agt $\in \mathcal{A}$ and $q \in Q$, that is, NOOP is always available to all agents and the system remains still when all agents perform it.

- Capability function $\Theta: \mathcal{C} \mapsto \mathcal{F}\left(\boldsymbol{\Pi}_{\text {Act }}^{\mathcal{P}}\right)$ maps capability terms to their (finite) set of plans. (Here, $\mathcal{F}(X)$ denotes the set of all finite subsets $X$.)

Example 2. Figure 1(a) shows a partial model for the gold game. The game starts at state $q_{0}$, with players $\mathrm{Ag}$ and En located at $B$ and $A$, resp., and gold present at $C$. From there, player Ag has a winning strategy: reach the gold earlier and deposit it in the depot. This can be seen in path $q_{0} q_{1} q_{2} q_{3} q_{4}$. However, this is possible only when the agent $\mathrm{Ag}$ is indeed equipped with all three capabilities. If, on the other hand, the agent lacks capability Collect, for instance, then player En may actually manage to win the game, as evident from the path $q_{0} q_{1} q_{5} q_{6} q_{7} q_{8}$.

BDI-ATLES models are similar to ATLES ones, except that capability, rather than strategy term, interpretations are used. In a nutshell, the challenge thus is to characterize what the underlying "low-level" ATL strategies for agents with certain capabilities and goals are. We call such strategies rational strategies, in that they are compatible with the standard BDI rational execution model [18]: they represent the agent acting as per her available plans in order to achieve her goals in the context of her beliefs.

So, given an agent agt $\in \mathcal{A}$, a plan-library $\Pi$, and a goal base $\mathcal{G}$, we define $\Sigma_{\Pi, \mathcal{G}}^{a g t}$ to be the set of standard ATL strategies for agent agt in $\mathcal{M}$ that are rational strategies when the agent is equipped with plan-library $\Pi$ and has $\mathcal{G}$ as (initial) goals, that is, those ATL strategies in which the agent always chooses an action that is directed by one of its available plans in order to achieve one of its goals in the context of its current beliefs. The core idea behind defining set $\Sigma_{\Pi, \mathcal{G}}^{a g t}$ is to identify those "rational traces" in the structure that are compatible with the BDI deliberation process in which the agent 
acts as per her goals and beliefs. Traces just generalize paths to account for the actions performed at each step, and are hence of the form $\lambda^{+}=q_{0} a_{1} q_{1} \cdots a_{\ell} q_{\ell}$ such that $q_{0} q_{q} \cdots q_{\ell}$ is a (finite) path. Rational strategies, then, are those that only yield rational traces. Technically, we define rational traces in three steps. First, we define a goalmarking function $g\left(\lambda^{+}, i\right)$ denoting the "active" goal base of the agent at the $i$-th stage of trace $\lambda^{+}$. Basically, a goal-marking function keeps track of the goals that the agent has already achieved at each stage in a trace. Second, we define $\operatorname{Exec}\left(\phi[\alpha] \psi, g, \lambda^{+}\right)$ as the set of indexes (i.e., stages) in trace $\lambda^{+}$where the plan $\phi[\alpha] \psi$ may have been executed by the agent: the plan's precondition $\phi$ was true, $\psi$ was an active goal of the agent (as directed by goal-marking function $g$ ), and $\alpha$ was indeed performed. Finally, we say a trace $\lambda^{+}$is deemed "rational" if at every moment in the run the agent executed one of its plans. That is, for every index $i$, it is the case that $i \in \operatorname{Exec}_{a g t}\left(\phi[\alpha] \psi, g, \lambda^{+}\right)$, for some plan $\phi[\alpha] \psi$ in her know-how library. Finally, we use $\Sigma_{\Pi, \mathcal{G}}^{a g t}$ to denote the set of all ATL strategies whose executions always yield rational traces. The laborious, and arguably boring, technical details of all the above steps and notions can be found in [21].

Example 3. Figure 1(b) depicts two possible traces $\lambda_{1}^{+}$and $\lambda_{2}^{+}$(for agent Ag) compatible with strategies $f_{\mathrm{Ag}}^{1}$ and $f_{\mathrm{Ag}}^{2}$, resp. Trace $\lambda_{1}^{+}$is due to the agent executing actions as per its applicable plans, as evident from the plan labeling. For example, at state $q_{1}$, the agent is in a gold location and hence executes the pick action as per plan $\mathrm{Ag}_{C} \wedge G_{C}[\mathrm{PICK}] G_{B}$. Consequently the strategy $f_{\mathrm{Ag}}^{1}$ is rational, as it yields rational trace $\lambda_{1}^{+}$. Trace $\lambda_{2}^{+}$on the other hand does not obey the BDI rationality constraints (e.g., the agent remains still in location $B$, despite an applicable plan being available).

Assuming that set $\Sigma_{\Pi, \mathcal{G}}^{a g t}$ of rational strategies has been suitably defined, we are ready to detail the semantics for formulas of the form $\langle\langle A\rangle\rangle_{\omega, \varrho} \varphi$. Following ATLES we first extend the notion of a joint strategy for a coalition to that of joint strategy under a given capability and goal assignment. So, given a capability (goal) assignment $\omega(\varrho)$

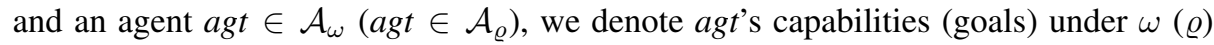
by $\omega[$ agt $](\varrho[$ agt $])$. Intuitively, an $\langle\omega, \varrho\rangle$-strategy for coalition $A$ is a joint strategy for $A$ such that $(i)$ agents in $A \cap \mathcal{A}_{\omega}$ only follow "rational" (plan-goal compatible) strategies as per their $\omega$-capabilities and $\varrho$-goals; and $(b)$ agents in $A \backslash \mathcal{A}_{\omega}$ follow arbitrary strategies. Formally, an $\langle\omega, \varrho\rangle$-strategy for coalition $A$ (with $\mathcal{A}_{\omega}=\mathcal{A}_{\varrho}$ ) is a collective strategy $F_{A}$ for agents $A$ such that for all $f_{\text {agt }} \in F_{A}$ with agt $\in A \cap \mathcal{A}_{\omega}$, it is the case that $f_{a g t} \in \Sigma_{I, \mathcal{G}}^{a g t}$, where $\Pi=\cup_{c \in \omega[a g t]} \Theta(c)$ and $\mathcal{G}=\varrho[a g t]$. Note no requirements are asked on the strategies for the remaining agents $A \backslash \mathcal{A}_{\omega}$, besides of course being legal (ATL) strategies. Also, whereas ATLES $\rho$-strategies are collective strategies including all agents in the domain of commitment function $\rho$, our $\langle\omega, \varrho\rangle$-strategies are collective strategies for the coalition of concern only. This is because commitment functions induce deterministic agent behaviors, whereas capabilities and goals assignments induce non-deterministic ones. We will elaborate on this issue below.

Using the notions of $\langle\omega, \varrho\rangle$-strategies and that of possible outcomes for a given collective strategy from ATL (refer to function out $(\cdot, \cdot)$ from Preliminaries), we are now able to state the meaning of BDI-ATLES (coalition) formulas: ${ }^{2}$

\footnotetext{
${ }^{2}$ As with ATL(ES), $\varphi$ ought to be a path formula and is interpreted in the usual manner. We omit the other ATL-like cases for brevity; see [20].
} 
$\mathcal{M}, q \models\langle\langle A\rangle\rangle_{\omega, \varrho} \varphi$ iff there is a $\langle\omega, \varrho\rangle$-strategy $F_{A}$ such that for all $\langle\omega, \varrho\rangle$-strategies $F_{\mathcal{A}_{\omega} \backslash A}$ for $\mathcal{A}_{\omega} \backslash A$, it is the case that $\mathcal{M}, \lambda \models \varphi$, for all paths $\lambda \in \operatorname{out}\left(q, F_{A} \cup F_{\mathcal{A}_{\omega} \backslash A}\right)$.

Intuitively, $F_{A}$ stands for the collective strategy of agents $A$ guaranteeing the satisfaction of formula $\varphi$. Because $F_{A}$ is a $\langle\omega, \varrho\rangle$-strategy, some agents in $A$-those whose capabilities and goals are defined by $\omega$ and $\varrho$, resp.- are to follow strategies that correspond to rational executions of its capabilities. At the same time, because other agents outside the coalition could have also been assigned capabilities and goals, the chosen collective strategy $F_{A}$ needs to work no matter how such agents (namely, agents $\mathcal{A}_{\omega} \backslash A$ ) behave, as long as they do it rationally given their plans and goals. That is, $F_{A}$ has to work with any rational collective strategy $F_{\mathcal{A}_{\omega} \backslash A}$. Finally, the behavior of all remaining agents-namely those in $\mathcal{A} \backslash\left(A \cup \mathcal{A}_{\omega}\right)$-are taken into account when considering all possible outcomes, after all strategies for agents in $A \cup \mathcal{A}_{\omega}$ have been settled.

While similar to ATLES coalition formulas $\langle\langle A\rangle\rangle_{\rho} \varphi$, BDI-ATLES coalition formulas $\left\langle\langle A\rangle_{\omega, \varrho} \varphi\right.$ differ in one important aspect that makes its semantics more involved. Specifically, whereas commitment functions $\rho$ prescribe deterministic behaviors for agents, capabilities and goals assignments yield multiple potential behaviors for the agents of interest. This nondeterministic behavior stems from the fact that BDI agents can choose what goals to work on at each point and what available plans to use for achieving such goals. Technically, this is reflected in the strategies for each agent in $\left(\mathcal{A}_{\omega} \backslash A\right)$-those agents with assigned capabilities and goals but not part of the coalition - cannot be (existentially) considered together with those of agents in $A$ or (universally) accounted for via the possible outcomes function out $(\cdot, \cdot)$, as such function puts no rationality constraints on the remaining (non-committed) agents. Thus, whereas agents in $A \cap \mathcal{A}_{\omega}$ are allowed to select one possible rational behavior, all rational behaviors for agents in $\left(A_{\omega} \backslash A\right)$ need to be taken into consideration.

We close this section by noting an important, and expected, monotonicity property of BDI-ATLES w.r.t. changes in the goals and plans of agents.

Proposition 1. $\models\langle\langle A\rangle\rangle_{\omega, \varrho} \varphi \supset\left\langle\left\langle A^{\prime}\right\rangle\right\rangle_{\omega^{\prime}, \varrho^{\prime}} \varphi$ holds, provided that:

- $A \subseteq A^{\prime}$, that is, the coalition is not reduced;

- $\omega[$ agt $] \subseteq \omega^{\prime}[$ agt $]$ and $\varrho[$ agt $] \subseteq \varrho^{\prime}[$ agt $]$, for all agt $\in \mathcal{A}_{\omega} \cap A$, that is, the goals and capabilities of those BDI agents in the coalition are not reduced; and

- $\mathcal{A}_{\omega} \backslash A \subseteq \mathcal{A}_{\omega^{\prime}} \backslash A^{\prime}$, that is, the set of non BDI agents outside the coalition is not reduced (but could be new BDI agents outside the coalition);

- $\omega^{\prime}[$ agt $] \subseteq \omega[$ agt $]$ and $\varrho^{\prime}[$ agt $] \subseteq \varrho[$ agt $]$, for all agt $\in \mathcal{A}_{\omega} \backslash A$, that is, the goals and capabilities of those BDI agents outside the coalition are not augmented.

Informally, augmenting the goals/plans of agents in a coalition does not reduce the ability of agents. This is because a collective $\langle\omega, \varrho\rangle$-strategy for coalition $A$ to bring about a formula would still work if more goals and plans are given to the agents in the coalition (second condition). Observe, on the other hand, that augmenting the goals or plans of those agents outside the coalition may yield new behavior that can indeed interfere with the coalition's original abilities (last condition). This even includes turning BDI agents into non BDI agents (third condition). Of course, as in ATL, enlarging the coalition does not reduce ability (first condition). 


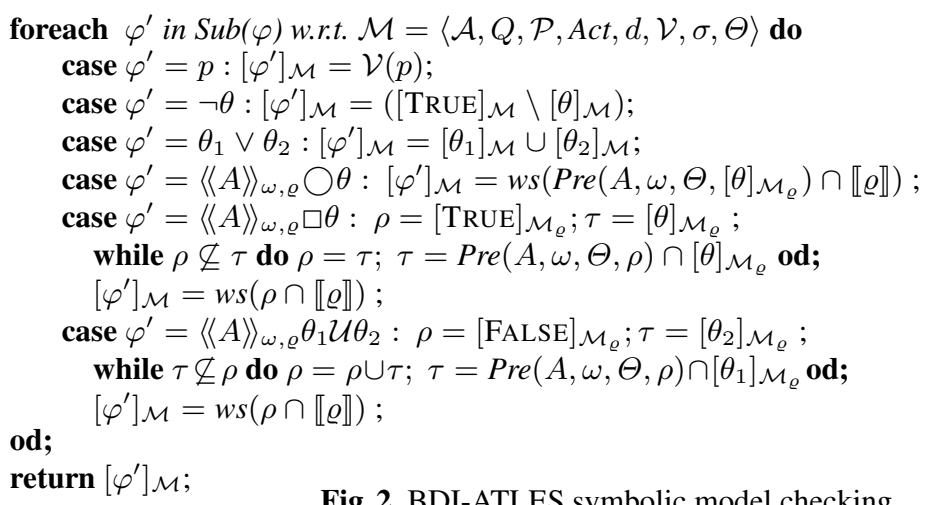

\section{BDI-ATLES Model Checking}

Given a BDI-ATLES model $\mathcal{M}$ and a formula $\varphi$, the model checking algorithm for BDI-ATLES computes the set of states in $\mathcal{M}$ that satisfy $\varphi$. To that end, the algorithm has to take into account the rational choices of each BDI agent, that is, those choices that are the consequence of the agent's goals and capabilities specified by functions $\varrho$ and $\omega$ in formulae of the form $\left\langle\langle A\rangle_{\omega, \varrho} \varphi\right.$. Roughly speaking, the algorithm restricts, at each step, the options of BDI agents to their applicable plans. We start by extending the model $\mathcal{M}$ to embed the possible goals (based on the goal assignment) of BDI agents into each state, and then then discuss the model checking algorithm and its complexity.

So, given a BDI-ATLES model $\mathcal{M}=\langle\mathcal{A}, Q, \mathcal{P}, A c t, d, \mathcal{V}, \sigma, \Theta\rangle$ and a goal assignment $\varrho$, the goal-extended model is a model $\mathcal{M}_{\varrho}=\left\langle\mathcal{A}, Q_{\varrho}, \mathcal{P}\right.$, Act, $\left.d_{\varrho}, \mathcal{V}_{\varrho}, \sigma_{\varrho}, \Theta\right\rangle$, where:

- $Q_{\varrho} \subseteq Q \times \prod_{a g t \in \mathcal{A}_{\varrho}} 2^{\varrho[a g t]}$ is the set of extended states, now accounting for the possible goals of BDI agents. When $q_{\varrho}=\left\langle q, g_{1}, \ldots, g_{\left|\mathcal{A}_{\varrho}\right|}\right\rangle \in Q_{\varrho}$, where $q \in Q$ and $g_{i} \subseteq \varrho\left[a g t_{i}\right]$, is an extended state, we use $w s\left(q_{\varrho}\right)=q$ and $g l\left(a g t_{i}, q_{\varrho}\right)=g_{i}$ to project $\mathcal{M}$ 's world state and $a g t_{i}$ 's goals. To enforce belief-goal consistency we require no agent ever wants something already true: there are no $q_{\varrho} \in Q_{\varrho}$, agt $\in \mathcal{A}_{\varrho}$, and formula $\gamma$ such that $\mathcal{V}\left(w s\left(q_{\varrho}\right)\right) \models \gamma$ and $\gamma \in \operatorname{gl}\left(\right.$ agt,$\left.q_{\varrho}\right)$.

- $\mathcal{V}_{\varrho}\left(q_{\varrho}\right)=\mathcal{V}\left(w s\left(q_{\varrho}\right)\right)$, for all $q_{\varrho} \in Q_{\varrho}$, that is, state evaluation remains unchanged.

- $d_{\varrho}\left(a g t, q_{\varrho}\right)=d\left(a g t, w s\left(q_{\varrho}\right)\right)$, that is, physical executability remains unchanged.

- $\sigma_{\varrho}\left(q_{\varrho}, a\right)=\left\langle q^{\prime}, g_{1}^{\prime}, \ldots, g_{\left|\mathcal{A}_{\varrho}\right|}^{\prime}\right\rangle$, where $q^{\prime}=\sigma\left(w s\left(q_{\varrho}\right), a\right)$ and $g_{i}^{\prime}=g l\left(a g t_{i}, q_{\varrho}\right) \backslash$ $\left\{\gamma \mid \gamma \in g l\left(a g t_{i}, q_{\varrho}\right), \mathcal{V}\left(q^{\prime}\right) \models \gamma\right\}$, is the transition function for the extended model.

Model $\mathcal{M}_{\varrho}$ is like $\mathcal{M}$ though suitably extended to account for agents' goals under the initial goal-assignment $\varrho$. Observe that the transition relation caters for persistence of goals as well as dropping of achieved goals. Indeed, the extended system will never evolve to an (extended) state in which some agent has some true fact as a goal. Hence, the transition relation is well-defined within $Q_{\varrho}$ states. More interesting, the extended model keeps the original physical executability of actions and, as a result, it accommodates both rational and irrational paths. However, it is now possible to discriminate between them, as one can reason about applicable plans in each state. Finally, it is not 
difficult to see that the extended model is, in general, exponentially larger than the original one with respect to the number of goals $\max _{\text {agt }} \in \mathcal{A}(\mid \varrho[$ agt $] \mid)$ and agents $\left|\mathcal{A}_{\varrho}\right|$.

As standard, we denote the states satisfying a formula $\varphi$ by $[\varphi]$. When the model is not clear from the context, we use $[\varphi]_{\mathcal{M}}$ to denote the states in $\mathcal{M}$ that satisfy the formula $\varphi$. We extend $w s(\cdot)$ projection function to sets of extended states in the straightforward sense, that is, ws $(S)=\bigcup_{q \in S}\{w s(q)\}$. Thus, ws $\left([\varphi]_{\mathcal{M}_{\varrho}}\right)$ denotes the set of all world states in $\mathcal{M}$ that are part of an extended state in $\mathcal{M}_{\varrho}$ satisfying the formula $\varphi$. Also, $\varrho \rrbracket$ denotes the set of extended states where the agents' goals are as per goal assignment $\varrho$; formally, $\llbracket \varrho \rrbracket=\left\{q \mid q \in Q_{\varrho}, \forall a g t \in \mathcal{A}_{\varrho}: \operatorname{gl}(\right.$ agt,$q)=\varrho[$ agt $\left.]\right\}$.

Figure 2 shows the model checking algorithm for BDI-ATLES. It is based on the symbolic model checking algorithm for ATL [3] and ATLES [20]. The first three cases are handled in the same way as in ATL(ES). To check the BDI-ATLES coalition formulae $\langle\langle A\rangle\rangle_{\omega, \varrho} \varphi$, we extend the model as above (relative to the formula's goal assignment $\varrho)$, and then check the plain ATL coalition formula $\langle\langle A\rangle\rangle \varphi$ in such extended model. Note that only the set of states having the goals as per the initial goal assignment are returned-all agents' initial goals are active in the first state of any rational trace.

Unlike standard ATL model checking, we restrict the agents' action choices as per their capabilities. This is achieved by modifying the usual pre-image function Pre (.) to only take into account actions resultant from agents' applicable plans. More concretely, $\operatorname{Pre}(A, \omega, \Theta, \rho)$ is the set of (extended) states from where agents in coalition $A$ can jointly force the next (extended) state to be in set $\rho$ no matter how all other agents (i.e., agents in $\mathcal{A} \backslash A$ ) may act and provided all BDI-style agents (i.e., agents with capabilities defined under $\omega$ and $\Theta$ ) behave as such. Formally:

$$
\begin{aligned}
\operatorname{Pre}(A, \omega, \Theta, \rho)=\left\{q \mid \forall i \in A, \exists a_{i} \in d_{\varrho}^{+}(i, q, \omega, \Theta),\right. \\
\left.\quad \forall j \in \mathcal{A} \backslash A, \forall a_{j} \in d_{\varrho}^{+}(j, q, \omega, \Theta): \sigma_{\varrho}\left(q,\left\langle a_{1}, \ldots, a_{|\mathcal{A}|}\right\rangle\right) \in \rho\right\},
\end{aligned}
$$

where auxiliary function $d_{\varrho}^{+}($agt $, q, \omega, \Theta)$ denotes the set of all actions that an agent agt may take in state $q$ under capabilities as per defined in $\omega$ and $\Theta$ :

$$
d_{\varrho}^{+}(\text {agt }, q, \omega, \Theta)= \begin{cases}d_{\varrho}(\text { agt }, q) & \text { if agt } \notin \mathcal{A}_{\omega} \\ d_{\varrho}(\text { agt }, q) \cap d^{\mathrm{BDI}}\left(\text { agt }, q, \bigcup_{c \in \omega[\text { agt }]} \Theta(c)\right) & \text { if agt } \in \mathcal{A}_{\omega}\end{cases}
$$

An action belongs to set $d_{\varrho}^{+}(a g t, q, \omega, \Theta)$ if it is physically possible (i.e., it belongs to $d_{\varrho}(a g t, q)$ ), and BDI-rational whenever the agent in question is a BDI agent. To capture the latter constraint, set $d^{\mathrm{BDI}}(a g t, q, \Pi)$ is defined as the set of all rational actions for agent agt in (extended) state $q$ when the agent is equipped with the set of plans $\Pi$ :

$$
d^{\mathrm{BDI}}(\text { agt }, q, \Pi)= \begin{cases}\{a \mid \phi[a] \psi \in \Delta(\text { agt }, q, \Pi)\} & \text { if } \Delta(\text { agt }, q, \Pi) \neq \emptyset \\ \{\mathrm{NOOP}\} & \text { otherwise }\end{cases}
$$

where $\Delta($ agt $, q, \Pi)=\{\phi[a] \psi \in \Pi \mid \mathcal{V}(q) \models \phi, \gamma \in \operatorname{gl}($ agt,$q), \psi \models \gamma\}$ is the set of all applicable plans in $\Pi$ at state $q$. So, summarising, function $\operatorname{Pre}(\cdot, \cdot, \cdot, \cdot)$ is an extension of the standard ATL Pre $(\cdot)$ function in which the agents that have goals and capabilities defined - the BDI agents-do act according to those goals and capabilities. 
It is clear that the modified version of $\operatorname{Pre}(\cdot)$ function does not alter the complexity of the underlying ATL-based algorithm. In fact, the variation is similar to that used for model checking ATLES, except that the action filtering does not come from strategy terms, but from agent plans. This means that the algorithm runs in polynomial time w.r.t. the size of model $\mathcal{M}_{\varrho}($ which is exponential w.r.t. the original model $\mathcal{M})$.

Theorem 1. Model checking a BDI-ATLES formula $\langle\langle A\rangle\rangle_{\omega, \varrho} \varphi$ (against a model $\mathcal{M}$ ) can be done in exponential time on the number of agents $|\mathcal{A}|$ and goals $\max _{a \in \mathcal{A}}(|\varrho[a]|)$.

Of course, should we have included agents' goals explicitly in models (rather than using a succinct representation), as done with intentions in ATL+intentions (ATLI) [15], the model checking problem would retain ATL's polynomial complexity. The same would apply if one just generalized ATLES to explicitly require all rational-strategies be part of the model. The fact is, however, that generating such rational strategies by hand (to include them in models) will be extremely involved, even for small problems. In addition, our approach decouples agent's mental attitudes from the physical ATL-like model, and enables reasoning at the level of formulae without changing the model.

We shall note that the exponentiality may not show up in certain applications. In many cases, for example, one is interested in just one BDI agent acting in an environment. In that case, only such agent will be ascribed goals and capabilities. Since it arises due to agents with goals, the exponential complexity would therefore only be on the number of goals for such agent. Similarly, in situations where all agents have a single goal to achieve (e.g., to pick gold), the model checking would then be exponential on the number of BDI agents only. In the next section we shall provide one interpretation of goals for which the model checking problem remains polynomial.

\section{BDI-ATLES with Maintenance Goals}

So far, we have worked on the assumption that agents have a set of "flat" achievement goals, goals that the agent needs to eventually bring about. One can however consider alternative views of goals that could suit different domains. In particular, we have considered achievement goals with priorities and repetitive/reactive maintenance goals. In the first case, the framework can be easily generalized to one in which goals can be prioritized without an increase in complexity [21].

A more promising case arises when goals are given a maintenance interpretation, that is, (safety) properties that ought to be preserved temporally. For example, a Mars robot has the goal to always maintain the fuel level above certain threshold. We focus our attention on the so-called repetitive or reactive maintenance goals [10, 12]: goals that ought to be restored whenever "violated." Should the fuel level drop below the threshold, the robot will act towards re-fueling. This type of goals contrast with proactive maintenance goals [12], under which the agent is expected to proactively avoid situations that will violate the goal. The fact is, however, that almost all BDI platformlike JACK, JASON, and JADEX — only deal with the reactive version, thus providing a middle ground between expressivity and tractability.

Technically, to accommodate maintenance goals within BDI-ATLES, one only needs to do a small adaptation of the semantics of the logic so that goals are not 
dropped forever once satisfied, but "re-appear" when violated. We refer to this alternate version of our logic as BDI-ATLES ${ }^{M}$. Of course, the model checking algorithm discussed above also needs to be slightly adapted to deal with the new goal semantics. Interestingly, one only needs to adapt the definition of a goal-extended model $M_{\varrho}$ by re-defining components $Q_{\varrho}$ and $\sigma_{\varrho}\left(q_{\varrho}, a\right)$; see [21] for details.

Theorem 2. Model checking in BDI-ATLES ${ }^{M}$ can be done in polynomial time (w.r.t. the model and the formula).

Hence, for (reactive) maintenance goals, we retain ATL(ES) polynomial complexity. ${ }^{3}$ Of course, this bound is tight, as BDI-ATLES ${ }^{M}$ subsumes ATL (just take $\omega=\varphi=$ $\emptyset$ in every coalition formula) and model checking ATL is PTIME-complete [3].

\section{Discussion}

We have developed an ATL-like logic that relates closely to the BDI agent-oriented programming paradigm widely used to implement multi-agent systems. In the new logic, the user can express the capability of agents equipped with know-how knowledge in a natural way and can reason in the language about what agents can achieve under such capabilities. Besides the general framework with standard achievement goals, we argued that one could instead appeal to goals with priorities or a special type of maintenance goals. We provided algorithms for model checking in such a framework and proved its (upper-bound) complexity in the various cases. Overall, we believe that this work is a first principled step to bring together two different fields in the area of multiagent systems, namely, verification of strategic behaviour and agent programming.

The framework presented here made a number of assumptions requiring further work. Due to valuation function $\mathcal{V}$ in a structure, all agents are assumed to have full shared observability of the environment. This is, of course, a strong assumption in many settings. We considered here basically reactive plans, akin to the language of GOAL [11], certain classes of 2APL/3APL [8, 13], reactive modules [4], and universal plans [19]. We would like to explore the impact of allowing plan bodies having sequences of actions, and more importantly, sub-goaling, as well as the possibility of agents imposing (new) goals to other agents, via so-called BDI messages. Also, in the context of complex plan bodies, one could then consider both a linear as well as interleaved execution styles of plans within each agent (for its various goals). Most of these issue appear to be orthogonal to each other, and hence can be investigated one by one. With the core framework laid down, our next efforts shal focus on the above issues, as well as proving whether the complexity result provided in Theorem 1 is tight.

We close by noting that, besides ATLES, our work has strong similarities and motivations to those on plausibility [14] and intention [15] reasoning in ATL. Like ATLES, however, those works are still not linked to any approach for the actual development of agents, which is the main motivation behind our work. Nonetheless, we would like to investigate how to integrate plausibility reasoning in our logic, as it seems orthogonal to that of rational BDI-style behavior. Indeed, the plausibility approach allows us to focus the reasoning to certain parts of an ATL structure using more declarative specifications.

\footnotetext{
${ }^{3}$ Note the complexity of model checking ATLES is known only for memoryless strategies [20].
} 


\section{Bibliography}

[1] N. Alechina, M. Dastani, B. Logan, and J.-J. Meyer. A logic of agent programs. In Proc. of the National Conference on Artificial Intelligence (AAAI), pages 795-800, 2007.

[2] N. Alechina, M. Dastani, B. Logan, and J.-J. Meyer. Reasoning about agent deliberation. In Proc. of Principles of Knowledge Representation and Reasoning (KR), pages 16-26, 2008.

[3] R. Alur, T. A. Henzinger, and O. Kupferman. Alternating-time temporal logic. Journal of the ACM, (49):672-713, 2002.

[4] C. Baral and T. C. Son. Relating theories of actions and reactive control. Electronic Transactions of AI (ETAI), 2(3-4):211-271, 1998.

[5] R. H. Bordini, L. Braubach, M. Dastani, A. Fallah-Seghrouchni, J. J. Gómez Sanz, J. Leite, G. O'Hare, A. Pokahr, and A. Ricci. A survey of programming languages and platforms for multi-agent systems. Informatica (Slovenia), 30(1):33-44, 2006.

[6] M. E. Bratman, D. J. Israel, and M. E. Pollack. Plans and resource-bounded practical reasoning. Computational Intelligence, 4(3):349-355, 1988.

[7] P. Busetta, R. Rönnquist, A. Hodgson, and A. Lucas. JACK intelligent agents: Components for intelligent agents in Java. AgentLink Newsletter, 2:2-5, Jan. 1999.

[8] M. Dastani. 2APL: A practical agent programming language. Autonomous Agents and Multi-Agent Systems, 16(3):214-248, June 2008.

[9] M. Dastani and W. Jamroga. Reasoning about strategies of multi-agent programs. In Proc. of Autonomous Agents and Multi-Agent Systems (AAMAS), pages 997-1004, 2010.

[10] M. Dastani, B. van Riemsdijk, and J.-J. Meyer. Goal types in agent programming. In Proc. of Autonomous Agents and Multi-Agent Systems (AAMAS), pages 1285-1287, 2006.

[11] F. de Boer, K. Hindriks, W. van der Hoek, and J. Meyer. A verification framework for agent programming with declarative goals. Journal of Applied Logic, 5(2):277-302, 2007.

[12] S. Duff, J. Harland, and J. Thangarajah. On proactivity and maintenance goals. In Proc. of Autonomous Agents and Multi-Agent Systems (AAMAS), pages 1033-1040, 2006.

[13] K. V. Hindriks, F. S. de Boer, W. van der Hoek, and J.-J. Meyer. Agent programming in 3APL. Autonomous Agents and Multi-Agent Systems, 2(4):357-401, 1999.

[14] W. Jamroga and N. Bulling. A framework for reasoning about rational agents. In Proc. of Autonomous Agents and Multi-Agent Systems (AAMAS), pages 1-3, 2007.

[15] W. Jamroga, W. Van Der Hoek, and M. Wooldridge. Intentions and strategies in game-like scenarios. Progress in Artificial Intelligence, pages 512-523, 2005.

[16] L. Padgham and P. Lambrix. Formalisations of capabilities for BDI-agents. Autonomous Agents and Multi-Agent Systems, 10(3):249-271, May 2005.

[17] A. S. Rao and M. P. Georgeff. Modeling rational agents within a BDI-architecture. In Proc. of Principles of Knowledge Representation and Reasoning (KR), pages 473-484, 1991.

[18] A. S. Rao and M. P. Georgeff. An abstract architecture for rational agents. In Proc. of Principles of Knowledge Representation and Reasoning (KR), pages 438-449, 1992.

[19] M. Schoppers. Universal plans for reactive robots in unpredictable environments. In Proc. of the Int. Joint Conference on Artificial Intelligence (IJCAI), pages 1039-1046, 1987.

[20] D. Walther, W. van der Hoek, and M. Wooldridge. Alternating-time temporal logic with explicit strategies. In Conference on Theoretical Aspects of Rationality and Knowledge, pages 269-278. ACM Press, 2007. doi: 10.1145/1324249.1324285.

[21] N. Yadav and S. Sardina. Reasoning about agent programs using ATL-like logics. Available from CoRR http: //arxiv.org/abs/1207.3874, 2012. 